\title{
Noncontact electrical resistivity measurement technique for molten metals
}

\author{
Won-Kyu Rhim ${ }^{\text {a) }}$ and Takehiko Ishikawa ${ }^{\text {b) }}$ \\ Jet Propulsion Laboratory, California Institute of Technology, Pasadena, California 91109
}

(Received 21 April 1998; accepted for publication 14 July 1998)

\begin{abstract}
A noncontact technique of measuring the changes in electrical conductivity (or resistivity) of conducting liquids is reported. The technique is based on a conducting drop that is levitated by the high-temperature electrostatic levitator in a high vacuum. This technique, which utilizes the principle of the asynchronous induction motor, measures the relative changes in torque as a function of temperature by applying a rotating magnetic field to the sample. Changes in electrical resistivity are related to the changes in measured torque using the formula developed for the induction motor. Validity of this technique was demonstrated using a pure aluminum sample around its melting temperature. When the measurement results were calibrated by a literature value of resistivity at the melting point, our resistivity data around the melting point could be expressed by $r_{\text {liq }}=24.19$ $+1.306 \times 10^{-2}\left(T-T_{m}\right) \mu \Omega \mathrm{cm}$ over $T_{m} \sim 1160 \mathrm{~K}, r_{\text {solid }}=10.77+1.421 \times 10^{-2}\left(T-T_{m}\right) \mu \Omega \mathrm{cm}$ over $700 \mathrm{~K} \sim T_{m}$, and the thermal conductivity as determined by the Wiedemann-Franz-Lorenz law from the resistivity data was given by $\kappa_{\text {liq }}(T)=94.61+4.41 \times 10^{-2}\left(T-T_{m}\right) \mathrm{W} \mathrm{m}^{-1} \mathrm{~K}^{-1}$, $\kappa_{\text {solid }}(T)=211.13-7.57 \times 10^{-2}\left(T-T_{m}\right) \quad \mathrm{W} \mathrm{m}^{-1} \mathrm{~K}^{-1}$. Both electrical resistivity and thermal conductivity are in close agreement with the literature, confirming the validity of the present technique. (c) 1998 American Institute of Physics. [S0034-6748(98)03510-2]
\end{abstract}

\section{INTRODUCTION}

Electrical conductivity is one of the most sensitive indicators of changes in the nature of the chemical binding. In general, the electrical conductivity is proportional to the carrier density and the carrier mobility. A change in the nature of the chemical binding primarily alters the carrier density, and the structural changes alter the carrier mobility. Very early investigation of metals showed that conductivity decreases approximately by a factor of 2 at the melting point, while it increases in silicon and germanium as they transform from semiconducting solids to conducting liquids. Electrical conductivity plays an important role in technical applications: it controls the flow of melts under the influence of electromagnetic force in the process of refining or growing semiconductor crystals, and it is a sensitive measure of concentration fluctuation in a critically mixed liquid alloy near the critical point in the homogeneous liquid phase.

In a conventional rotating magnetic-field method for electrical conductivity, a cylindrical container holding a molten liquid is suspended by a tungsten wire, and the torque experienced by the hanging cylinder is measured when a rotating magnetic field is applied. ${ }^{1}$ However, chemically reactive liquids, especially molten refractory materials, contained in the cylinder may be easily contaminated, altering intrinsic electrical properties. Furthermore, one cannot expect those liquids to reach a deeply undercooled state as long as the container provides heterogeneous nucleants that trigger early solid-phase nucleations.

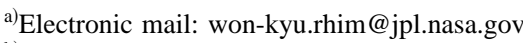

${ }^{b}$ On leave from the National Space Development Agency of Japan. Present Address: Space Utilization Research Center, NASDA, 2-1-1 Sengen Tsukuba Ibaraki 305 Japan.
}

In this article, we present a noncontact electrical conductivity measurement technique that was developed for the high-temperature electrostatic levitator (HTESL). ${ }^{2}$ Noncontact processing of refractory materials using the HTESL will allow us to investigate properties of molten liquids without contamination both in their superheated as well as undercooled liquid states. Since a melt in the HTESL is well isolated from container walls through a high vacuum, the melt will maintain its intrinsic properties, providing opportunities to investigate the structures and properties of deeply undercooled liquid states. However, special caution may be required to correct for the changing sample mass when processing a material having high vapor pressure.

Also demonstrated in this article is the determination of thermal conductivity of aluminum using the measured electrical resistivity data. Accurate measurement of thermal conductivity of molten metals is usually more difficult than the measurement of electrical conductivity. Inaccuracies in directly measured thermal conductivities are primarily caused by the difficulties in accurate heat flow measurements, and also to a certain degree by flows taking place in a melt. However, if thermal and electric conductions take place primarily by free electrons, these two conductivities are connected through the Wiedemann-Franz-Lorenz law. Use of this law may allow us to obtain more accurate values for thermal conductivity from accurately measured electrical conductivity.

\section{EXPERIMENTAL APPARATUS AND APPROACH}

The high-temperature electrostatic levitator levitates a sample 1-3 $\mathrm{mm}$ in diameter between a pair of parallel disk electrodes (Fig. 1). The electric field between these two electrodes generates an electrostatic force on a charged sample to 

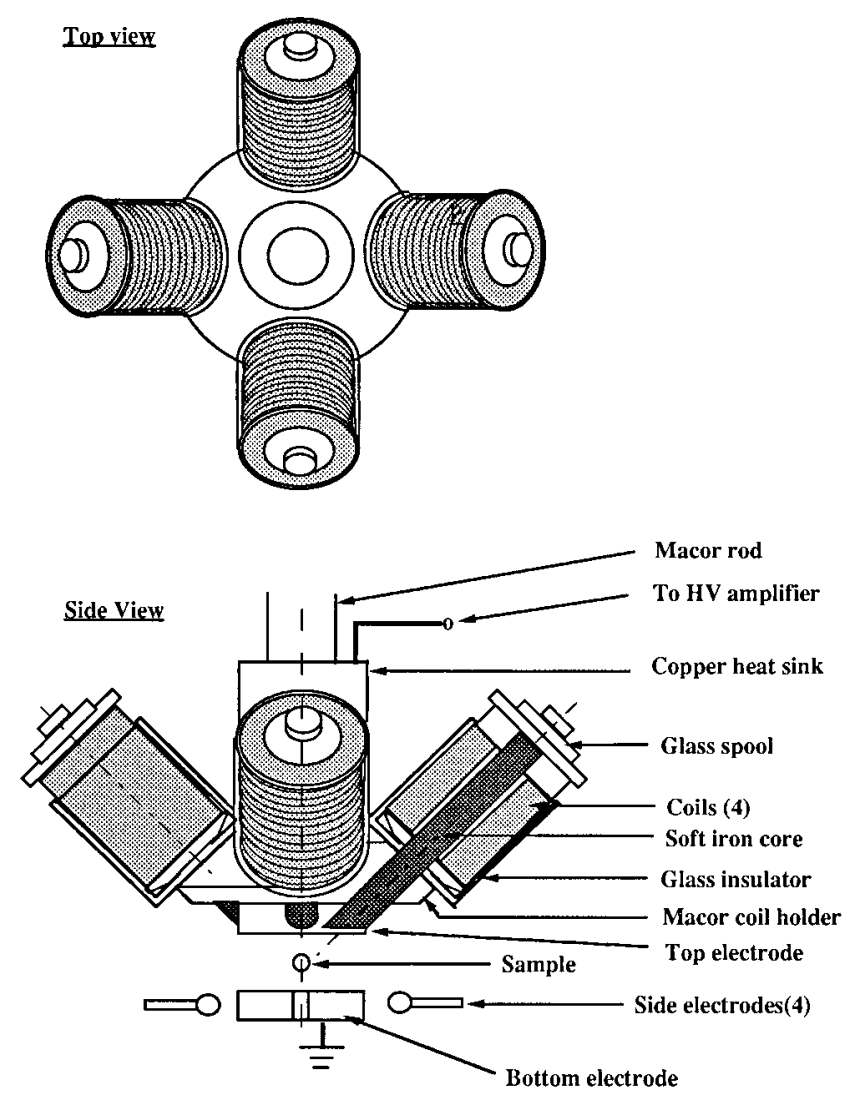

FIG. 1. Top and side views of the electrode assembly with rotation coils designed for the high-temperature electrostatic levitator at JPL. A charged conducting sample levitated between the electrodes can be rotated around the vertical direction when a rotating magnetic field is applied around the same direction.

cancel the gravitational force, and the four small side electrodes around the bottom electrode control the sample position along the horizontal direction. The four coils positioned around the top electrode produce a horizontal magnetic field that rotates at an appropriate frequency in order to induce sample rotation around the vertical axis. The electrode assembly is housed by a stainless-steel chamber, which is typically evacuated to $10^{-8}$ Torr before sample heating begins. A schematic diagram of the levitation chamber is shown in Fig. 2. A 1-kW UV-rich high-pressure xenon arc lamp is used for the sample heating. The sample temperature was measured by a single color pyrometer $\left(E^{2} T\right)$ operating at a wave length longer than $4 \mu \mathrm{m}$ in order to isolate it from the xenon lamp spectrum. A detailed description of the HTESL is given in an earlier publication. ${ }^{2}$

Four identical coils were wound. Each coil was wound on a glass spool ( $28 \mathrm{~mm}$ in length, and $\sim 8 \mathrm{~mm}$ in diameter) with 28-gauge insulated copper wire. Spools were made out of silica glass for electrical insulation purposes, since the coils were to be mounted in close proximity of the highvoltage electrode (the top electrode). When 400 turns were wound on a spool, it showed $\sim 34 \mathrm{mH}$ and $\sim 5.3 \Omega$. When a soft iron core ( $0.25 \mathrm{in}$. in diameter, and $2.25 \mathrm{in}$. in length) was slid through each coil, approximately $3.2 \mathrm{mT}$ of magnetic field was detectable at an end of the core when the coil carried $140 \mathrm{~mA}$. As shown in Fig. 1, these four coils were mounted on the top electrode with iron cores deeply imbed-

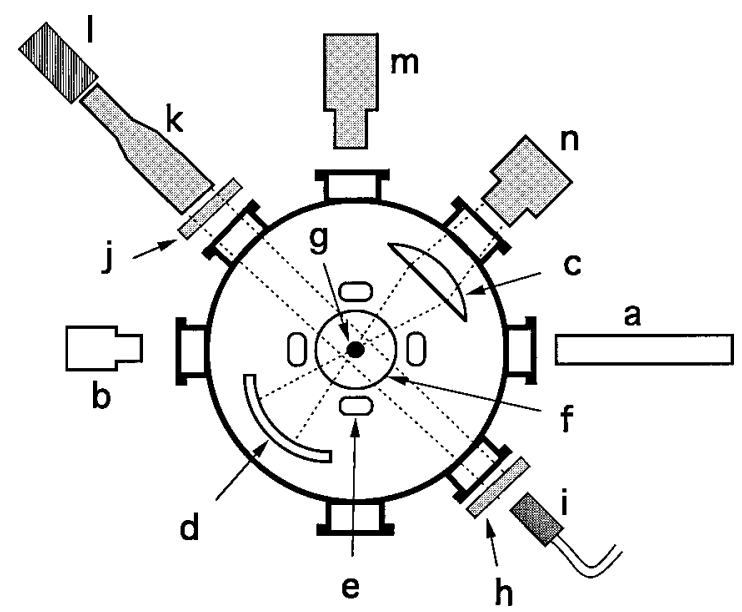

FIG. 2. Schematic diagram of the high-temperature electrostatic levitator at JPL: (a) HeNe position sensing laser, (b) position sensing detector, (c) focusing lens, (d) focusing reflector, (e) side positioning electrode, (f) top/ bottom electrode, (g) sample, (h) back light diffuser, (i) fiber optical back light, (j) He-Ne blocking filter, (k) long distance microscope, (l) CCD camera, $\mathrm{m}$ pyrometer, $\mathrm{n}$ and xenon heating lamp.

ded in it to maximize the magnetic field experienced by a levitated sample.

The four-coil assembly so mounted was driven by a current-producing electronic assembly as shown in Fig. 3. A dual-channel signal generator (HP 3326A) produces two sinusoidal signals with $90^{\circ}$ phase difference. We found that $400 \mathrm{~Hz}$ was adequate frequency for the present experiment. These two signals were then amplified by two separate amplifiers (HP 6825A) before they were connected to a switch box. This switch box does the traffic control of currents which are going through each coil to produce various magnetic fields. This switch has the following four settings: (1) an OFF switch cuts off all currents through the coils, (ii) a CLOCKWISE switch wires the coils so that the horizontal magnetic field seen by the sample rotates in the clockwise direction, (iii) C-CLOCKWISE reverses the field rotation direction, and (iv) an additional switch not shown in Fig. 3 is a VERTICAL switch which connects the coils in such a way as to produce a magnetic field along the vertical direction at the sample position. This VERTICAL switch is built-in so that it can damp the sample rotation if its rotational axis is other than in the vertical direction. The CLOCKWISE or the C-CLOCKWISE modes can be used either to accelerate or decelerate the sample, depending upon its current rotational state. The currents flowing through each set of coils are monitored by measuring voltages across a $1-\Omega$ resistor.

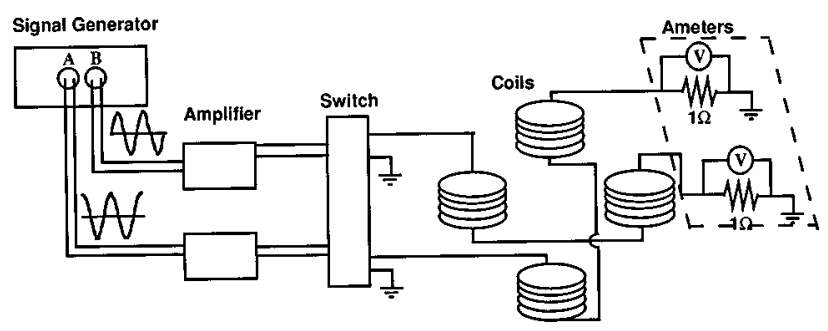

FIG. 3. A schematic diagram of an electronics assembly that generates and channels appropriate electric currents to the coils to produce the desired magnetic field at the sample position. 
Accurate measurement of sample rotation frequency is important for accurate determination of torque imparted to the sample at different instantaneous rotation frequencies. In the present experiment, a $\mathrm{He}-\mathrm{Ne}$ laser beam was directed to the sample, and the reflected beam was detected by a silicon photodetector. The output voltage of the detector was amplified and digitized to get the Fourier power spectrum using a microcomputer. Such a power spectrum showed peaks at all harmonics of the sample rotation frequency. An additional method which was used in parallel with the spectrum method was to utilize the strobe effect created by a TV monitor. Regular monitors run at the $30-\mathrm{Hz}$ frame rate (or at the $60-\mathrm{Hz}$ field rate). A charge-coupled device (CCD) camera running at a fast shutter speed was mounted on a telemicroscope to produce a magnified sample image on a TV screen. Whenever the sample rotation rate approached one of the harmonic or subharmonic frequencies of the $60-\mathrm{Hz}$ field rate, the sample seemed to slow down to show a completely static image at the harmonic/subharmonic frequency. Identification of $15,20,30,40,45,60,75 \mathrm{~Hz}, \ldots$, of the sample rotation rate could be done precisely (within $0.01 \mathrm{~Hz}$ ). Such a stroboscopic approach assisted by the power spectrum display allowed unambiguous determination of sample rotation frequency. When the drop shape was axisymmetric, the methods described above can be applied only when there is some distinguishable surface structure on the drop. On the surface of the molten aluminum drop used in the present experiment, several aluminum oxide patches floating on the drop surface served as indicators. If drops do not show any surface structures, they should be spun above respective bifurcation points where they will turn into a triaxial spheroidal shape. Such bifurcation phenomena have been well established by both theoretical analysis ${ }^{3}$ as well as experimental confirmations. ${ }^{4-7}$ While staying in the triaxial region of a rotating drop, the same detection method described above can be applied. Again, small changes in the moment of inertia, which are caused by the deformation over a frequency interval, have to be detected, and its effect should be accounted for in the torque analysis. The effect on the coupling of a drop to the magnetic field due to the shape deformation may be made negligible by limiting the deformation over a narrow frequency interval.

\section{EXPERIMENTAL PROCEDURE AND RESULTS}

The basic principle of the present sample rotation mechanism is essentially the same as the asynchronous induction motor. The four-coil assembly works as the stator while the levitated sample acts as the rotor. According to the principle of the induction motor, ${ }^{8,9}$ if an ac voltage $E_{1}$ at frequency $\omega_{s}$ is applied to a stator having resistance $R_{1}$ and inductance $L_{1}$, the torque $\tau$ experienced by the rotor (having its own resistance $R_{2}$ and inductance $L_{2}$, which is rotating at an instantaneous frequency $\omega$, is given by

$$
\tau=\frac{\omega_{s} E_{1}^{2}}{R_{1}^{2}+\omega_{s}^{2} L_{1}^{2}}\left(\frac{s R_{2}}{R_{2}^{2}+s^{2} L_{2}^{2}}\right),
$$

where

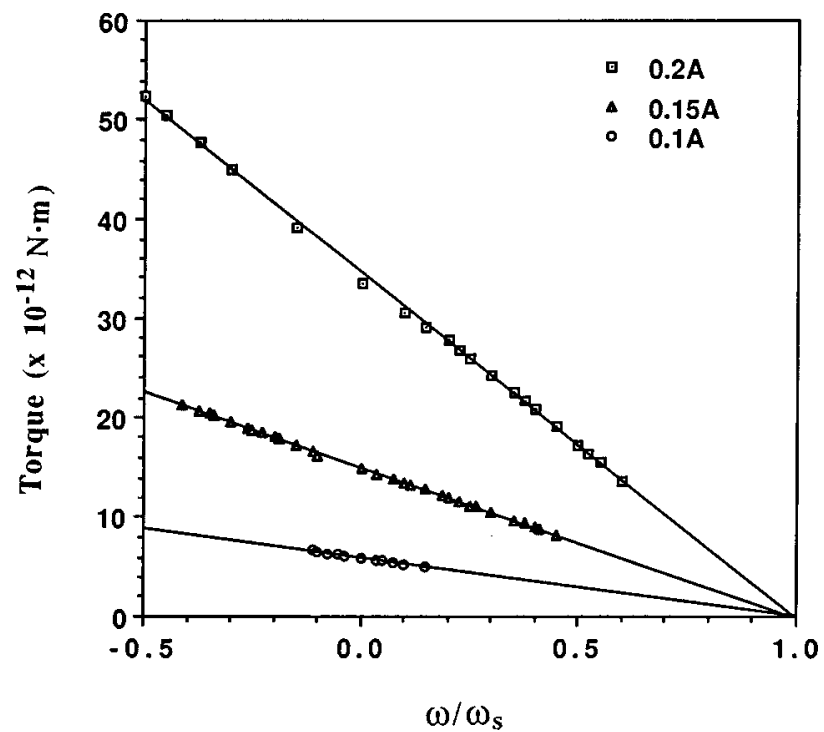

FIG. 4. Linear relationship between the measured torque on a levitated aluminum sphere $(2.38 \mathrm{~mm}$ in diameter $)$ and its instantaneous rotation frequency for three different values of coil current. The rotation frequency of the applied magnetic field was $400 \mathrm{~Hz}$.

$$
s \equiv \frac{\omega_{s}-\omega}{\omega_{s}} .
$$

It is clear from Eq. (1) that in order to measure the resistivity of a rotor it is important to keep $E_{1}$ and $\omega_{s}$ constant throughout the measurement process. In this experiment, this requirement was satisfied by maintaining the stator currents constant. Equation (1) was tested by levitating an aluminum sphere and measuring torque as a function of instantaneous sample rotation frequency. It can be seen in Fig. 4 that at a fixed stator current, the torque is a linear function of the instantaneous sample rotational frequency. This means that the relationship $R_{2}^{2} \gg s^{2} L^{2}$ is well satisfied in Eq. (1), transforming the equation to a simpler form:

$$
\tau \approx\left(\frac{\omega_{s} E_{1}^{2}}{R_{1}^{2}+\omega_{s}^{2} L_{1}^{2}}\right) \frac{1}{R_{2}}\left(1-\frac{\omega}{\omega_{s}}\right) .
$$

This equation shows that when the left parenthesis (the stator term) is kept constant, the measured torque decreases linearly as a function of the rotor frequency, and its gradient is inversely proportional to the resistance of the rotor. Figure 4 shows the results obtained at three different coil (stator) currents. If the stator current is kept constant, any changes in the slope of this line must be related to changes in the electrical resistance of the rotor. In the absence of any ferromagnetic materials (those soft iron cores in the present case), the measured torque should be proportional to the square of the current. As can be seen in Fig. 4, the proportionality between the torque and the square of the coil current holds only approximately. However, it is worth emphasizing that the present technique relies heavily on the linear relationship between the torque and the instantaneous rotor frequency when the stator term is fixed.

Once the validity of Eq. (3) was demonstrated, using a solid aluminum sphere, we are ready to apply it on a molten material whose resistivity at its melting temperature is well 

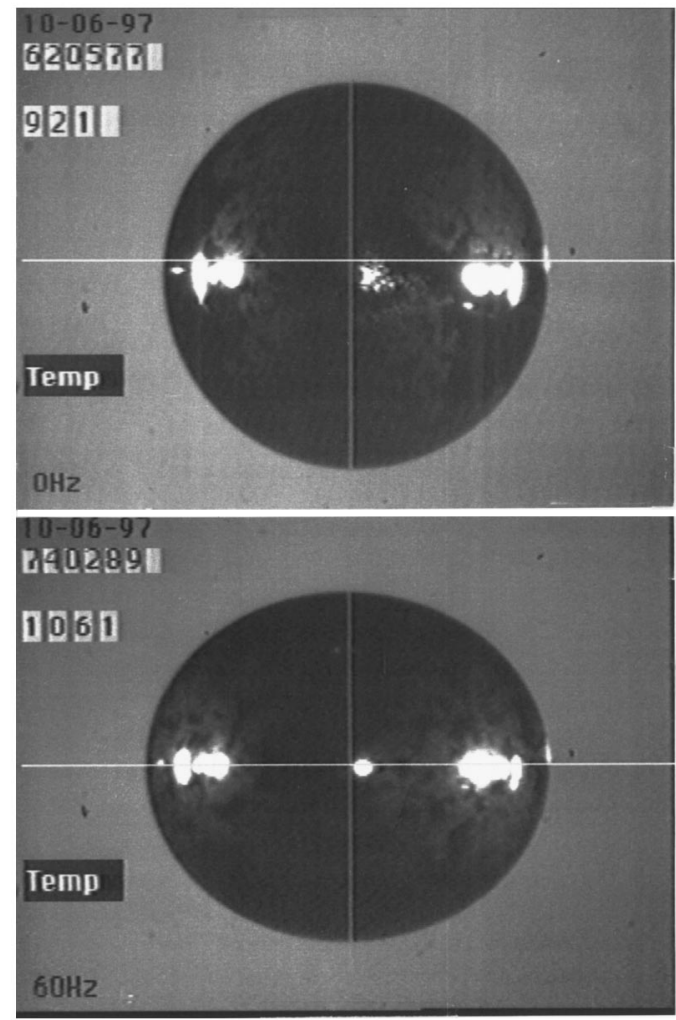

FIG. 5. Side views of a levitated aluminum drop showing a spherical shape when it is static (top picture), and an oblate spheroid when it is rotating at the rate of $60 \mathrm{~Hz}$ around the vertical axis (bottom picture).

known. Pure aluminum was chosen for such a material. The sample material was heated above its melting point while it is levitated in the HTESL. For reasons of accurate detection of the sample rotation frequency, the average torque of the drop rotation was measured at frequencies between 20 and $30 \mathrm{~Hz}$, and back to $20 \mathrm{~Hz}$ by timing each interval, instead of measuring the torque on the sample in the neighborhood of $\omega=0$. This is justified as long as the linearity between torque and rotational frequency holds. According to Eq. (3), when the frequency of the applied rotating field is $400 \mathrm{~Hz}$, the wind-up torque is given by

$$
\tau_{+}=\frac{C}{R_{2}}\left(1-\frac{25}{400}\right),
$$

and the wind-down torque is given by

$$
\tau_{-}=\frac{C}{R_{2}}\left(1+\frac{25}{400}\right)
$$

giving the average torque to be

$$
\bar{\tau}=\frac{\tau_{+}+\tau_{-}}{2}=\frac{C}{R_{2}},
$$

which is identical to the torque measured at $0 \mathrm{~Hz}$. In the above equations, $C$ represents the stator term shown in Eq. (3). Since the average torque exerted on a sample that changed its rotation frequency from $\omega_{1}$ to $\omega_{2}$ between the time $t_{1}$ and $t_{2}$ can be expressed by

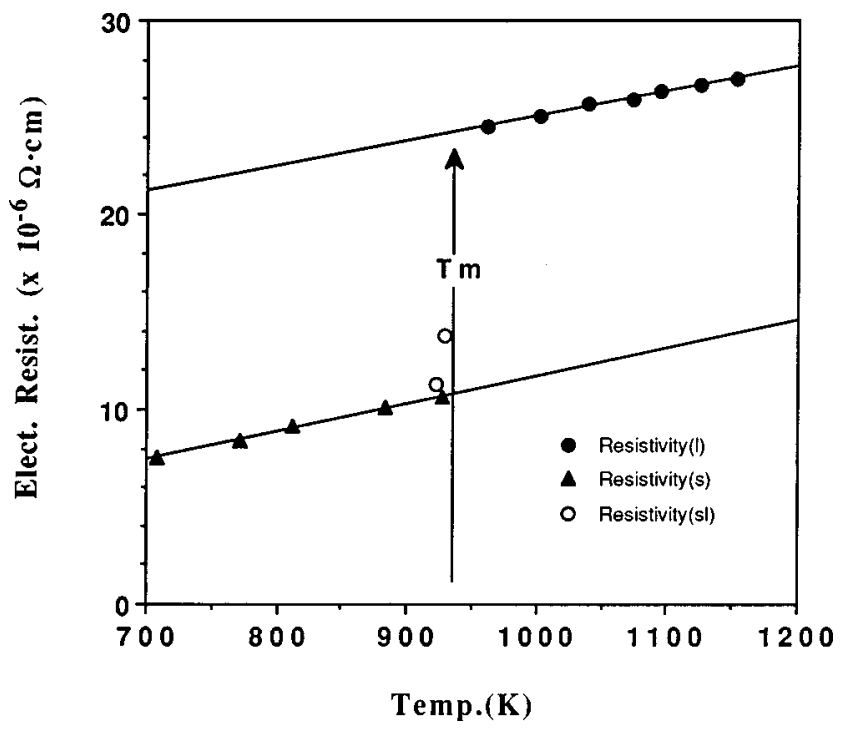

FIG. 6. Electrical resistivity of a pure aluminum sample measured as a function of temperature, using the present technique. The literature value of resistivity of molten aluminum at the melting temperature was used as a single calibration point to obtain this result. (s), (1), and (sl) in the figure designate the solid phase, the liquid phase, and the solid-to-liquid transition, respectively.

$$
\bar{\tau}=\frac{I\left(\omega_{2}-\omega_{1}\right)}{t_{2}-t_{1}}=I \frac{\Delta \omega}{\Delta t},
$$

in which $I$ is the moment of inertia of the sample, a comparison with Eq. (6) shows that $R_{2}$ should be proportional to $\Delta t$ if $I \Delta \omega$ remains constant. Indeed, $\Delta \omega$ can remain fixed (at 10 $\mathrm{Hz}$, for example, in the present experiment). If we express with $(\Delta t)_{+}$the time interval measured as the molten aluminum drop was spun from 20 to $30 \mathrm{~Hz}$, and with $(\Delta t)_{-}$the time taken from $30 \mathrm{~Hz}$ back to $20 \mathrm{~Hz}$, the average torque can be expressed by

$$
\bar{\tau}=\frac{\tau_{+}+\tau_{-}}{2}=\frac{1}{2} I \Delta \omega \frac{(\Delta t)_{+}+(\Delta t)_{-}}{(\Delta t)_{+}(\Delta t)_{-}} .
$$

If we compare Eq. (6) with Eq. (8), at a fixed $I \Delta \omega, R_{2}$ should be proportional to the measured time intervals by

$$
R_{2}=\frac{2 C}{I \Delta \omega} \frac{(\Delta t)_{+}(\Delta t)_{-}}{(\Delta t)_{+}+(\Delta t)_{-}} .
$$

$(\Delta t)_{+}$and $(\Delta t)_{-}$were measured at different sample temperatures both above and below the melting point. Also recorded at each temperature were the drop images (at 20 and $30 \mathrm{~Hz}$ ), which were used to calibrate the final resistivity results due to the changing moment of inertia. The moment of inertia showed a slight change as the sample shape changed at a different rotation frequency and sample temperature (see Fig. 5). Such changes in moment of inertia have to be taken into account in deriving the final result. When the time interval data so obtained by Eq. (9) around the melting temperature $\left(T_{m}=933.452 \mathrm{~K}\right)$ was calibrated by the resistivity of molten aluminum at the melting point $(24.185 \mu \Omega \mathrm{cm})$ given by a reference value, ${ }^{1}$ the resistivity data obtained at different temperatures are as shown in Fig. 6. The solid lines are the linear fits to the data for the solid and the liquid branches, which can be expressed by

$$
r_{\text {liq }}=24.19+1.306 \times 10^{-2}\left(T-T_{m}\right) \mu \Omega \mathrm{cm},
$$


while the expression by Iida and Guthrie ${ }^{1}$ is given by

$$
r_{\text {liq }}=24.19+1.45 \times 10^{-2}\left(T-T_{m}\right) \mu \Omega \mathrm{cm} .
$$

Since Iida's resistivity value at $T=T_{m}=933.452 \mathrm{~K}$ was used as the reference point, the first terms in each expression should be same. However, our temperature dependence of resistivity, $d r_{\text {liq }} / d T$, is approximately $10 \%$ smaller than that of Iida's. For the resistivity of the solid phase below $T_{m}$, our result can be expressed by

$$
r_{\text {solid }}=10.77+1.421 \times 10^{-2}\left(T-T_{m}\right) \mu \Omega \mathrm{cm} .
$$

The only literature data we could find on the resistivity of solid aluminum near the melting point was $r_{\text {solid }}\left(T_{m}\right)$ $=10.9,{ }^{1}$ which is in close agreement with our result. It is worth noting that the $d r_{\text {solid }} / d T$ we obtained near $T_{m}$ is substantially different from that of the room-temperature value, $0.429 \times 10^{-2} \mu \Omega \mathrm{cm} / \mathrm{K}$, given in the literature. ${ }^{10}$

Since both solid and molten aluminum are conductors where free electrons are responsible for the electrical and thermal conductivities, one can use the Wiedemann-FranzLorenz law to relate the thermal conductivity to the electrical resistivity:

$$
\frac{\kappa_{\text {liq }} r_{\text {liq }}}{T}=\frac{\pi^{2} k^{2}}{3 e^{2}} \equiv L_{0}=2.45 \times 10^{-8} \mathrm{~W} \Omega \mathrm{K}^{-2},
$$

where $k$ is the Boltzman constant and $e$ is the electron charge. The constant $L_{0}=\pi^{2} k^{2} / 3 e^{2}$ is the Lorenz number whose validity was experimentally confirmed with high accuracy by Busch et al. ${ }^{11}$

Using the Eqs. (10), (12), and (13), one can obtain the following expressions for the thermal conductivities of solid and molten aluminum around $T_{m}$ :

$$
\begin{aligned}
& \kappa_{\text {liq }}(T)=94.61+4.41 \times 10^{-2}\left(T-T_{m}\right) \\
& \mathrm{W} \mathrm{m}^{-1} \mathrm{~K}^{-1} \text { over } T_{m} \sim 1160 \mathrm{~K}, \\
& \kappa_{\text {solid }}(T)=211.13-7.57 \times 10^{-2}\left(T-T_{m}\right) \\
& \mathrm{W} \mathrm{m}^{-1} \mathrm{~K}^{-1} \text { over } 700 \mathrm{~K} \sim T_{m} .
\end{aligned}
$$

These results are shown in Fig. 7 along with several other values from the literature. ${ }^{12-15}$ The agreement between our results with independently measured thermal conductivities given by the literature are quite good.

\section{DISCUSSION}

In this article we described a noncontact technique that measures relative changes of electrical conductivity of molten (or solid) metals. It, therefore, requires reference data for calibration. To the best of our knowledge, this is probably the first attempt of its kinds which can be applied to levitated melts. Having been adapted to work on a melt levitated by the HTESL in a high vacuum, it may be applicable to measure electrical conductivity of highly reactive melts of refractory materials as well as deeply undercooled melts of glassforming materials. For liquids where reference data are not available, this technique may be extended in the future to use as a carefully selected standard sample for all calibration purposes.

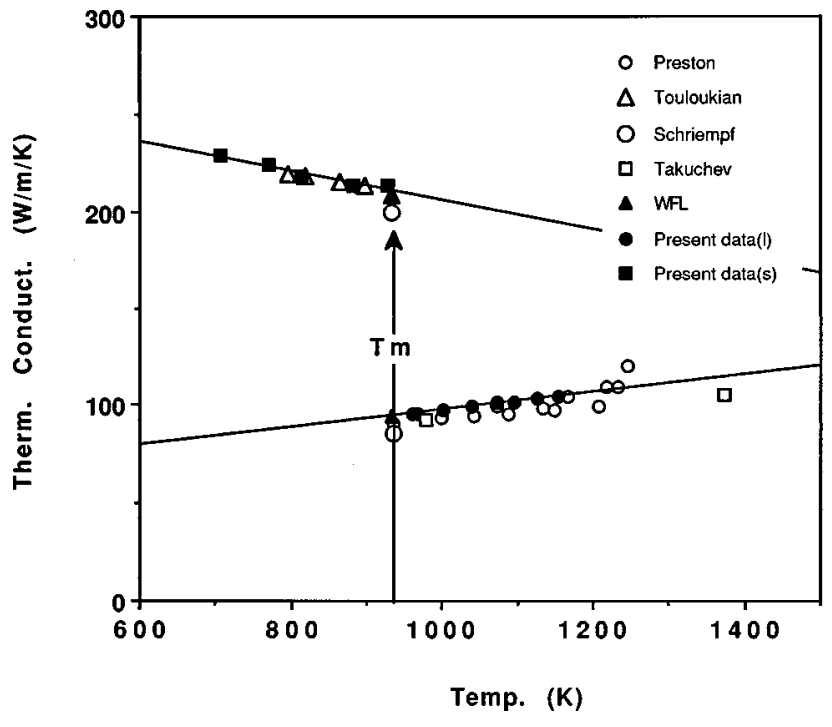

FIG. 7. Thermal conductivity of molten aluminum obtained from the results shown in Fig. 5 using the Wiedemann-Franz-Lorenz law, and other values in the literature for comparison purposes. (s) and (1) in the figure designate the solid phase and the liquid phase, respectively, and the solid lines indicate linear fits to the present data.

The Wiedemann-Franz-Lorenz law was used to determine the thermal conductivity of solid and liquid aluminum, showing good agreement with independently measured reference values. Such indirect measurement of thermal conductivity may prove to be important, particularly for low viscosity liquids where direct measurement of thermal conductivity is susceptible to errors caused by the convective flows within the liquids.

As an application of the present technique, direct measurement of electrical conductivity and indirect determination of thermal conductivity through the WiedemannFranz-Lorenz law were carried out on molten germanium with good results. These will be published elsewhere. ${ }^{16}$

\section{ACKNOWLEDGMENTS}

The authors would like to thank Daniel Barber and Dr. Paul-Francois Paradis for various assistance in this work. One of the authors (T.I.) appreciates NASDA's generous support during his one year stay at JPL to conduct this research. This work was carried out at the Jet Propulsion Laboratory, California Institute of Technology, under contract with the National Aeronautical and Space Administration.

${ }^{1}$ T. Iida and R. I. L. Guthrie, The Physical Properties of Liquid Metals (Clarendon, Oxford 1988), p. 228.

${ }^{2}$ W. K. Rhim, S. K. Chung, D. Barber, K. F. Man, G. Gutt, A. Rulison, and R. E. Spjut, Rev. Sci. Instrum. 64, 2961 (1993).

${ }^{3}$ R. A. Brown and L. E. Scriven, Proc. R. Soc. London, Ser. A 371, 331 (1980).

${ }^{4}$ W-K. Rhim, S. K. Chung, and D. D. Elleman, AIP Conf. Proc. 197, 91 (1988).

${ }^{5}$ A. Biswas, E. W. Leung, and E. H. Trinh, J. Acoust. Soc. Am. 90, 1502 (1991).

${ }^{6}$ T. G. Wang, A. V. Anilkumar, C. P. Lee, and K. C. Lin, J. Fluid Mech. 276, 389 (1994).

${ }^{7} \mathrm{~W}-\mathrm{K}$. Rhim and T. Ishikawa (unpublished).

${ }^{8}$ S. A. Nasar and I. Boldea, Electric Machines (Steady State Operation) (Hemisphere, New York, 1990). 
${ }^{9}$ P. L. Alger, Nature of Induction Machine (Gordon and Breach Science, New York, 1965).

${ }^{10}$ Handbook of Chemistry and Physics, 72 nd ed., edited by D. R. Lide, (CRC, Boca Raton, FL, 1992).

${ }^{11}$ G. Busch, H.-J. Guentherodt, W. Haller, and P. Wyssmann, Phys. Lett. 43A, 225 (1973).

${ }^{12}$ K. C. Mills, B. J. Monaghan, and B. J. Keene, Int. Mater. Rev. 41, 209 (1996).

${ }^{13}$ S. D. Preston and K. C. Mills, High Temp.-High Press. (to be published).
${ }^{14}$ Y. S. Touloukian, R. W. Powell, C. Y. Ho, and P. G. Klemens, Thermophysical Properties of Matter: Vol. 1 Thermal Conductivity of Metallic Elements and Allovs (IFI/Plenum, New York, 1970).

${ }^{15}$ J. T. Schriempf, High Temp.-High Press. 4, 411 (1972).

${ }^{16}$ W. K. Rhim and T. Ishikawa, "Thermophysical Properties of Molten Germanium Measured by the High Temperature Electrostatic Levitator," Proceedings of the 5th Asian Thermophysical Properties Conference, Seoul, Korea, August 30-September 2, 1998), pp. 611-616. 